\title{
On Bounded Part of an Algebra of Unbounded Operators
}

\author{
By
}

\author{
Subhash J. BHAтT*
}

\section{$\S 1$. Introduction}

By an $O p^{*}$-algebra $A$ is meant a collection of linear operators, not necessarily bounded, all defined in a dense subspace $D$ of a Hilbert Space $H$, satisfying $T D \subset D$ for all $T \in A$, which forms $a *$ algebra with vectorwise operations $(T+S) x=T x+S x, \quad(\lambda T) x=\lambda T x \quad(\lambda a$ scalar $),(T S) x=T(S x)$ and the involution $T \rightarrow T^{*}=\left.T^{\#}\right|_{D}, T^{\#}$ denoting the operator adjoint of $T$. It is also assumed that the identity operator $1 \in A$. The * subalgebra $A_{b}=\{T \in A \mid T$ is bounded $\}$ is the bounded part of $A$. Throughout, $\|\cdot\|$ denote operator norm on $A_{b}$.

$O p^{*}$-algebras have been investigated in the contect of quantum theory and representation theory of abstract (Non-Banach)* algebras, in particular, enveloping algebras of Lie algebras. Among selfadjoint $O p^{*}$-algebra [13], there are two classes that are better behaved viz. symmetric algebras ([5], [8], [9]) and countably dominated algebras ([2], [10], [11]). The objective of this paper is to examine role of $A_{b}$ in the structure of these two classes of algebras.

An $O p^{*}$-algebra $A$ is symmetric if for each $T \in A,\left(1+T^{*} T\right)^{-1}$ exists and $\left(1+T^{*} T\right)^{-1} \in A_{b}$. We prove the following that shows that in a symmetric algebra $A, A_{b}$ is very closely tied up with $A$, algebraically as well as topologically; and this infact characterizes symmetry.

Theorem 1. Let $A$ be an $O p^{*}$-algebra.

(a) If $A$ is symmetric, then $A_{b}$ is sequentially dense in $A$ in any * algebra topology $\tau$ on $A$ such that $B_{0}=\left\{T \in A_{b} \mid\|T\| \leqq 1\right\}$ is $\tau$-bounded.

(b) Let $\tau$ be any * algebra topology on $A$ such that the multiplication in $A$ is $\tau$-hypocontinuous, and $B_{0}$ is $\tau$-bounded and $\tau$-sequentially complete. If $\left(A_{b},\|\cdot\|\right)$ is sequentially $\tau$-dense in $A$, then $A$ is symmetric.

An $O p^{*}$-algebra $A$ is countably dominated if the positive cone $A^{+}$of $A$ contains a cofinal sequence $\left(A_{n}\right)$ in its natural ordering. We assume $A_{n} \geqq 1$ and

Communicated by H. Araki, January 11, 1988.

* Department of Mathematics, Sardar Patel University, Vallabh Vidyanagar 388 120, Gujarat, India. 
$A_{1}=1$. Additionally, $A_{n}^{-1} \in A$ for all $n$, then $A$ is said to satisfy condition (I) [2]. In non commutative integration with countably dominated algebras, significant role is being played by the $\sigma$-weak topology and $\rho$-topology (introduced in [3]) defined below.

For each $n$ define normed linear space $\left(n_{A_{n}}, \rho_{A_{n}}\right)$ by

$$
n_{A_{n}}=\left\{T \in A \mid \rho_{A_{n}}(T)=\sup _{x \in D} \frac{|\langle T x, x\rangle|}{\left\langle A_{n} x, x\right\rangle}<\infty\right\} .
$$

Then $A=\cup n_{A_{n}}$, and $\rho$-topology on $A$ is the inductive topology [14] defined by the embeddings $i d_{n}:\left(n_{A_{n}}, \rho_{A_{n}}\right) \rightarrow A$. Note that $A_{b}=n_{1}$. Now consider $\beta(D, D)$, the space of bounded sesquilinear forms on $D \times D$ with bibounded topology $\tau_{b b}$ defined by the seminorms $\beta \rightarrow \sup \left\{|\beta(x, y)| \mid x \in K_{1}, y \in K_{2}\right\}, K_{1}$ and $K_{2}$ varying over bounded subsets of $\left(D, t_{A}\right)$, where $t_{A}$ is the induced topology on $D$ defined by the seminorms $x \rightarrow\|T x\|(T \in A)$. (Note that $t_{A}$ is metrizable due to countable domination and $\left(D, t_{A}\right)$ can be assumed Frechet without loss of generality [11]). Then $\left(\beta(D, D), \tau_{b b}\right)$ is the strong dual of the Frechet Space $D \hat{\otimes} D$ (projective tensor product). The $\sigma$-weak topology on $A$ [2] is the relative topology in duced on $A$ by the weak topology $\sigma(\beta(D, D), D \hat{\otimes} D), A$ being naturally embedded in $\beta(D, D)$ by $T \rightarrow \beta^{T}:(x, y) \rightarrow\langle T x, y\rangle$. We also consider the following two other topologies, the first one defined completely in terms of the bounded part $A_{b}$ of $A$; and the other in terms of order structure on $A$.

(a) Dixon topology: Let $\Delta$ be the collection of all strictly positive functions on $A \times A$. For each $\delta \in \Delta$, let $N(\delta)=|c o| \cup\left\{\delta(S, T) S B_{0} T \mid S, T\right.$ in $\left.A\right\},|c o|$ denoting the absolutely convex hull. Let $\theta=\{N(\delta) \mid \delta \in \Delta\}$. Since $1 \in B_{0}$, each $N(\delta)$ is absorbing. Thus $\theta$ forms a 0 -neighbourhood base for a locally convex linear topology $I$ on $A$. It was considered first in [4] for a class of abstract topological $*$ algebras called generalized $B^{*}$-algebras which are realizable as $E C^{*}$-algebras [8] viz. symmetric $O p^{*}$-algebras $A$ with bounded part $A_{b}$ a $C^{*}$ algebra.

(b) Order topology: ([12], [14]): Let $\tau_{o h}$ be the order topology on $A^{b}=$ $\left\{T \in A \mid T=T^{*}\right\}$ viz. the largest locally convex linear topology making each order interval bounded. Let $\psi$ be a $\tau$-neighbourhood base for $\tau_{o h}$. For $U \in \psi$, let $\tilde{U}$ be the complex absolutely convex hull of $U$ in $A$. Then $\tilde{\psi}=\{\tilde{U} \mid U \in \phi\}$ is a $o$-neighbourhood base for a locally convex linear topology $\tau_{o}$ (complexification of $\tau_{o h}$ ).

We also prove the following.

Theorem 2. Let $A$ be a countably dominated $O p^{*}$-algebra satisfying condition (I). Then on $A$,

$$
\rho=\mathscr{I}=\tau_{b b}=\tau_{o}
$$

Proofs of both the theorems are presented in Part 3. In Part 2, we give a 
couple of lemmas that are needed and that appear to be of some independent interest. Finally the results are applied to countably dominated algebras.

\section{§ 2. Preliminary Lemmas}

Recall that in a topological * algebra $(A, t)$ (viz. a topological vector space with separately continuous multiplication and continuous involution), multiplication is called hypocontinuous if given a bounded set $B$ in $A$, and a 0 -neighbourhood $U$, there exists 0 -neighbourhoods $V_{1}$ and $V_{2}$ such that $B V_{1} \subset U, V_{2} B$ $\subset U$. The following is wellknown.

Lemma 2.1. In a topological algebra, joint continuity of multiplication implies hypocontinuity, and hypocontinuity of multiplication implies sequential joint continuity.

Lemma 2.2. Let $A$ be a countably dominated $O p^{*}$-algebra on a dense subspace $D$ of a Hilbert Space $H$. On $A_{b}=n_{1}$, the norm $\rho_{1}(\cdot)$ is equivalent to the operator norm $\|\cdot\|$.

Proof. For a $T \in A_{b}$,

$$
\begin{aligned}
\rho_{1}(T) & =\sup \{!\langle T x, x\rangle \mid: x \in D,\|x\|=1\} \\
& =\sup \{|\langle T x, x\rangle|: x \in H,\|x\|=1\} \\
& =w(T)
\end{aligned}
$$

where $w(T)$ is the numerical radius of $T$. By Halmos [7, p. 173], numerical radius defines a norm on $A_{b}$ equivalent to the operator norm; in fact, $1 / 2\|T\|$ $\leqq w(T) \leqq\|T\|$. Note that the validity of the lemma can also be alternatively seen by noting that for $T=T^{*}$ in $A_{b}, \rho_{1}(T)=\|T\|$, and so for any $T$ in $A_{b}$, $\rho_{1}(T) \leqq\|T\| \leqq 2 \rho_{1}(T)$.

$O p^{*}$-algebra $A$ is $\rho$-closed [3] if each $\left(n_{A_{n}}, \rho_{A_{n}}\right)$ is a Banach space. Also as discussed in [2, Proposition 5.1], in the presence of condition (I), there exists an onto isometric isomorphism $T^{(k)}:\left(n_{A_{k}}, \rho_{A_{k}}\right) \rightarrow\left(n_{1}, \rho_{1}\right)$. Hence the following is immediate.

Corollary 2.3. Let $A$ satisfies condition (I). Then $A$ is $\rho$-closed iff $\left(A_{b},\|\cdot\|\right)$ is a $C^{*}$-algebra.

The following lemma sheds some light on the role of $A_{b}$ in the structure of $A$.

Lemma 2.4. Let $A$ be an $O p^{*}$-algebra.

(a) If $B$ is a $\rho$-bounded * idempotent in $A$, then $B \subset B_{0}$. In particular, $B_{0}$ 
is closed in $\rho$.

(b) If $a *$ subalgebra $B$ of $A$ is a Banach *algebra under any norm $|$.$| such$ that $(B,||.) \rightarrow(A, \rho)$ is a continuous embeding, then $B$ consists of bounded operators.

(c) Let $A$ be $\rho$-closed. Let $T \in A, T \geqq 0$. If $n_{T}$ is an algebra, then $n_{T}$ consists of bounded operators; in particular, $T$ is bounded.

Proof. By Proposition 1.2 in [3], boundedness of $B$, together with the fact that $B$ is an idempotent, implies that there exists $S>0$ in $A$ such that $\left|\left\langle T^{n} x\right\rangle\right|$ $\leqq(S x, x)$ for all $x \in D, T \in B$, for all $n=1,2,3, \cdots \cdots$. Given $T \in B, Q=T^{*} T \in B$; and so for all $n$,

$$
\left\|Q^{n} x\right\|^{2}=\left\langle Q^{2 n} x, x\right\rangle \leqq\|x\|\left\|Q^{2 n} x\right\| .
$$

Hence iterating, for all $x \in D$, we obtain

$$
\begin{aligned}
\|Q x\| & \leqq\|x\|^{\left(1-1 / 2^{n}\right)}\left\|Q^{2^{n}} x\right\|^{1 / 2^{n}} \\
& \leqq\|x\|^{\left(1-1 / 2^{n}\right)}\langle S x, x\rangle^{1 / 2^{n}}
\end{aligned}
$$

for all $n$. This gives $\|Q x\| \leqq\|x\|$ for all $x$, and so $\|T x\| \leqq\|x\|$ for all $x$, showing $T$ to be bounded and $B \subset B_{0}$. (b) is immediate from (a); and (c) follows from (b).

Now for a locally convex linear topology $t$ on $A$, consider the following statements

(i) Involution in $A$ is $t$-continuous.

(ii) Multiplication in $A$ is separately $t$-continuous.

(iii) $B_{0}$ is $t$-closed.

(iv) $B_{0}$ is $t$-bounded.

(v) $B_{0}$ is the greatest member, under inclusion, of $\mathcal{B}^{*}(t)$ where $\mathcal{B}^{*}(t)$ is the collection of all absolutely convex, $t$-closed, $t$-bounded *idempotents in $A$.

The following describes basic properties of $\mathscr{I}$. This can be proved as in Dixon [4]. Part (d) is a consequence of Lemma 2.4(a).

Lemma 2.5. Let $A$ be an Op*-algebra

(a) I satisfies (i)-(v).

(b) I is finer than any locally convex topology $t$ satisfying (i)-(v).

(c) If $A_{b}$ is a $C^{*}$-algebra, then $\mathscr{I}$ is barrelled.

(d) I is finer than $\rho$.

\section{$\S 3$. Proofs of the Theorems}

Proof of theorem 1(a). Let $A$ be symmetric. Then for all $T \in A, \bar{T}^{*}=\bar{T}^{\#}$. This is a standard argument as in Lemma 2.1 in [8(I)] or Lemma 7.9 in [4]. 
Now given $T$ in $A$, take $T_{n}=T\left(1+(1 / n) T^{*} T\right)^{-1}$ for $n=1,2,3, \cdots \cdots$ Note that

$$
T_{1}=T\left(1+T^{*} T\right)^{-1}=T\left(1+\bar{T}^{*} \bar{T}\right)^{-1}=T\left(1+\bar{T}^{*} \bar{T}\right)^{-1}
$$

is in $A$ and is a bounded operator with $\left\|T_{1}\right\| \leqq 1$. Hence

$$
T_{n}=\sqrt{n}\left(\frac{T}{\sqrt{n}}\right)\left(1+\left(\frac{T}{\sqrt{n}}\right)^{*}\left(\frac{T}{\sqrt{n}}\right)\right)^{-1}
$$

is in $A_{b}$ with $\left\|T_{n}\right\| \leqq \sqrt{n}$. Further

$$
\begin{aligned}
T-T_{n} & =\frac{1}{n} T T^{*} T\left(1+\frac{1}{n} T^{*} T\right)^{-1} \\
& =\frac{1}{\sqrt{n}}\left(T T^{*}\right)\left(\frac{T}{\sqrt{n}}\right)\left(1+\left(\frac{T}{\sqrt{n}}\right)^{*}\left(\frac{T}{\sqrt{n}}\right)\right)^{-1}
\end{aligned}
$$

which is in $1 / \sqrt{n} T T^{*} B_{0}$. Let $\tau$ be any topology on $A$ such that $(A, \tau)$ is a topological *algebra. Let $B_{0}$ be $\tau$-bounded. Then given a 0 -neighbourhood $V$ in $A$, there exists a 0 -neighbourhood $U$ such that $T T * U \subset V$. Further, for sufficiently large $n, 1 / \sqrt{n} B_{0} \subset U$. Hence for such $n, T-T_{n} \in T T^{*} U \subset V$ showing that $T_{n} \rightarrow T$.

(b) Given $T$ in $A$, choose a sequence $T_{n}$ in $A_{b}$ such that $T_{n} \rightarrow T$ in $\tau$. Then $T_{n}^{*} \rightarrow T^{*}$, and so by Lemma $2.1, T_{n}^{*} T_{n} \rightarrow T^{*} T$. Let $S_{n}=\left(1+T_{n}^{*} T_{n}\right)^{-1}$ which are in $A_{b}$ with $\left\|S_{n}\right\| \leqq 1$. Now

$$
S_{n}-S_{m}=\left(1+T_{n}^{*} T_{n}\right)^{-1}\left(-T_{n}^{*} T_{n}+T_{m}^{*} T_{m}\right)\left(1+T_{m}^{*} T_{m}\right)^{-1} .
$$

By hypocontinuity, given a 0 -neighbourhood $U$, choose 0 -neighbourhoods $V_{1}$ and $V$ such that $B_{0} V_{1} \subset U ; V B_{0} \subset V_{1}$; with the result, $B_{0} V B_{0} \subset U$. Since $T_{n}^{*} T_{n}$ is Cauchy, $T_{m}^{*} T_{m}-T_{n}^{*} T_{n} \in V$ eventually. Hence $S_{n}-S_{m} \in B_{0} V B_{0} \subset U$ eventually. Thus the sequence $S_{n}$ in $B_{0}$ is Cauchy; hence $S_{n} \rightarrow S \in B_{0}$ by assumption. Then by sequential joint continuity of multiplication,

$$
\left(1+T^{*} T\right) S=\lim \left(1+T_{n}^{*} T_{n}\right) \quad\left(1+T_{n}^{*} T_{n}\right)^{-1}=1=S\left(1+T^{*} T\right) .
$$

Thus $S=\left(1+T^{*} T\right)^{-1} \in B_{0} \subset A_{0}$ showing that $A$ is symmetric.

Proof of Theorem 2. Note that as discussed in [2], $\rho=\tau_{b b}$ follows from the $\rho$-normality of the positive cone in $A$, a consequence of condition (I). We show $\tau_{0}=I$. Since $\tau_{0}$ is the largest locally convex linear topology on $A$ making each order interval in $A^{h}$ bounded, $\tau_{0} \geqq I$ follows if each order bounded set $M$ (which is contained in $\left[A_{n}, A_{n}\right]$ for some $\left.n\right)$ is $\mathcal{I}$-bounded, where $\left(A_{n}\right), A_{n} \geqq 1$ and $A_{n}^{-1} \in A$, is the sequence in $A^{+}$defined by condition (I). Thus for all $Z \in M$, $-A_{n}^{2} \leqq-A_{n} \leqq Z \leqq A_{n} \leqq A_{n}^{2}$; and so $-1 \leqq A_{n}^{-1} Z A_{n}^{-1} \leqq 1, A_{n}^{-1} Z A_{n}^{-1} \in B_{0}$. Now let $N(\delta)$ be $a$ and $\mathscr{I}$-neighbourhood of 0 . Then 
But

$$
N(\delta)=i c o \mid \cup\left\{\delta(X, Y) X B_{0} Y \mid X, Y \text { in } A\right\} \supseteqq \delta\left(A_{n}, A_{n}\right) A_{n} B_{0} A_{n}
$$

$$
\delta\left(A_{n}, A_{n}\right) Z=\delta\left(A_{n}, A_{n}\right) A_{n}\left(A_{n}^{-1} Z A_{n}^{-1}\right) A_{n} \in \delta\left(A_{n}, A_{n}\right) A_{n} B_{0} A_{n} \subset N(\delta) .
$$

Thus $M \subset\left(\delta\left(A_{n}, A_{n}\right)\right)^{-1} N(\delta)$ showing that $M$ is $\mathscr{I}$-bounded. Now to show $\tau_{0} \leqq \mathscr{I}$, we apply Lemma $2.5(\mathrm{~b})$. The topology $\tau_{0}$ is easily seen to satisfy (i) and (ii). Let $B \in \mathscr{B}^{*}\left(\tau_{0}\right)$. Then $B \mathscr{I}$-bounded as $\mathscr{I} \leqq \tau_{0}$; hence its $\mathscr{I}$-closure $\bar{B}$ is in $\mathcal{B}^{*}(\mathscr{I})$. Lemma 2.5(a) implies that $B \subset B_{0}$. The same argument applied to $B_{0}$ shows that $\tau_{0}$ satisfies (iii) and (iv); and so also (v). Thus $\tau_{0}=\mathscr{I}$; which, in view of Lemma $2.5(\mathrm{~d})$, gives $\tau_{0} \geqq \rho$. On the other hand, for each $n$, each bounded subset in the normed linear space $\left(n_{A_{n}}, \rho_{A_{n}}\right)$ is $\tau_{0}$-bounded. Hence the embedings $i d_{n}\left(n_{A_{n}}, \rho_{A_{n}}\right) \rightarrow\left(A, \tau_{0}\right)$ are continuous, $\left(n_{A_{n}}, \rho_{A_{n}}\right)$ being bornological. Since $\rho$ is the largest locally convex linear topology on $A$ making each of these embedings continuous, $\tau_{0} \leqq \rho$, and the proof is complete.

Corollary. Let $A$ be an $O p^{*}$-algebra.

(a) If $A$ is symmetric, then $A_{b}$ is sequentially dense in $(A, \rho)$

(b) Assume the following

(i) A satisfies condition (I)

(ii) The domain $D$ of $A$ is quasi-normable in the induced topology $t_{A}$.

(iii) $A_{b}$ is a $C^{*}$-algebra (in particular, $A$ is $\rho$-closed or $\sigma$-weakly closed). If $A_{b}$ is sequentially dense in $\rho$-topology, then $A$ is symmetric.

Proof. Theorem 1(a) gives (a). Note that if $A$ satisfies (I) and is $\sigma$-weakly closed, then $A_{b}$ is von Neumann algebra by [2]. Now (i) and (iii) in (b) together with Theorem 2 and Lemma 2.5(c) imply that $(A, \rho)$ is barelled, and in a barelled algebra, multiplication is known to be hypocontinuous. Further, due to quasinormability, $(A, \rho)$ satisfies strict condition of Mackey convergence [11]. From this, using Corollary 2.3 and Lemma 2.4(a), it is easily seen that $B_{0}$ is sequentially $\rho$-complete. Now the conclusion follows from Theorem 1(b).

Remarks. (a) Symmetry in an $O p^{*}$-algebra $A$ is a very stringent requirement, since as noted in the proof of Theorem $1(\mathrm{a}), \bar{T}^{*}=\bar{T}^{\#}$ holds, for all $T \in A$ which implies that in such an $A$, every hermitian element is essentially selfadjoint.

(b) Sequential density of $A_{b}$ in $(A, \rho)$ is not sufficient to make $A$ symmetric. As shown in $\left[11\right.$, Part 3], in the maximal $O p^{*}$-algebra $L(D)$ on Schwartz domain $D$, every $T \in A$ can be approximated, even in some normed space $\left(n_{A_{k}}, \rho_{A_{k}}\right)$, by a sequence of finite rank operators in $L(D)$. 


\section{Acknowledgements}

The author is very much thankful to the referee for constructive suggestions that led to a thorough revision of the paper and to removal of some discripencies.

\section{References}

[1] Allan, G. R., On a class of locally convex algebras, Proc. London Math-Soc. (3) 17 (1967), 91-114.

[2] Araki, H. and Jurzak, J., On a certain class of * algebras of unbounded operators, Publ. RIMS Kyoto Univ. 18 (1982), 1013-1044.

[3] Arnel, D. and Jurzak, J.P., Topological aspects of algebras of unbounded operators, Jr. Functional Analysis 24 (1979), 397-425.

[4] Dixon, P. G., Generalized $B^{*}$-algebras, Proc. London Math. Soc. (3) 21 (1970), 693-715.

[5] - Unbounded operator algebras, Proc. London Math. Soc. (3) 23 (1971), 5369.

[6] Grothendieck, A., Sur les espaces (F) et (DF), Summ. Brasil Math. 3 (1954), 57123.

[7 [ Halmos, P. R., A Hilbert Space Problem Book, Narosa Publ. House, New Delhi, 1978.

[8] Inoue, A., On a class of unbounded operator algebras I, II, III, IV, Pacific Jr. Math. 64 (1976), 77-98; ibid 66 (1976), 411-431; ibid 69 (1976), 105-133; Jr. Math. Anal. Appl. 64 (1978), 334-347.

[9] Unbounded generalizations of left Hilbert algebras, I, II, Jr. Functional Analysis 34 (1979), 339-362; ibid 35 (1980), 230-250.

[10] Jurzak, J.P., Unbounded Noncommutative Integration, D. Reidel Publ. Co., 1984.

[11] - Unbounded Operator algebras and DF spaces, Publ. RIMS Kyoto Univ., 17 (1981), 755-776.

[12] Kunze, W., Zur algebraischen and topologischen struktur der GC*-algebren, thesis, KMU, Leipzig, 1975.

[13] Powers, R. T., Self adjoint algebras of unbounded operators I, II, Comm. Math. Phys. 21 (1971), 88-124; Trans. American Math. Soc. 187 (1974).

[14] Schaeffer, H.H., Topological Vector Spaces, MacMillan, London 1966. 
